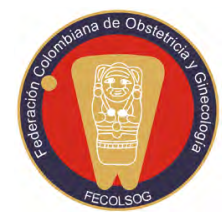

\title{
TRIBULACIONES DE LA INVESTIGACIÓN EN TIEMPOS DE COVID-19
}

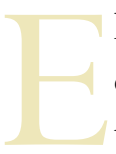

l año 2020 será recordado no solo por la aparición de una nueva pandemia (1), sino también por la gran incertidumbre que trajo al mundo ya que nos recordó la fragilidad del ser humano. Para inicios de este año, se consideraba que muy pocas situaciones eran imprevisibles o no pronosticables. Por ejemplo, a finales de 2017 se advertía que nos encontrábamos en un planeta globalizado, donde el cruce de personas, información y flujos de capital entre los países era cada vez más frecuente, llegando a niveles nunca vistos y, aunque había algunas amenazas - como la salida de la Gran Bretaña de la Unión Europea, conocida como Brexit, y el nacionalismo de Trump-, no se consideraba que hubiera una amenaza a esta globalización (2). Por otra parte, a partir del uso de la big data y de los modelos analíticos, que utilizan la llamada "inteligencia artificial”, se podían predecir muchas situaciones como posibles, por ejemplo, el comportamiento de las persona, ya fuera a partir de la visita a páginas de internet (3), o a partir de la ubicación del individuo a través del teléfono inteligente (4), o con el procesamiento de texto obtener inferencias causales (5), entre otras muchas a aplicaciones que estas tecnologías nos ofrecen. Muy pocas personas pudieron imaginar (6) que podría venir una pandemia por una infección viral, como la surgida en marzo de 2020, ante la cual estamos aún perplejos.

En Colombia, donde la incertidumbre es parte del escenario cotidiano, la pandemia nos sorprendió en un momento caracterizado por una gran movilización social, que se había iniciado en noviembre de 2019 como parte de una cadena de movimientos sociales en Latinoamérica (7), protestas que respondían a diferentes contextos y problemas, y que se pensaba iban a estar presentes durante todo el 2020 (8). Además, estaban presentes niveles muy altos de corrupción. Para 2019, el país estaba ubicado en el puesto 96 de 180, con un puntaje de 37/100 según Transparencia Internacional (9). El Estado colombiano ha tratado de contrarrestar la corrupción a través de normas, decretos, resoluciones y oficinas de control que llevan a procesos complejos que requieren, en condiciones de "normalidad", mucho tiempo para completar y que no han sido eficaces (10). Hay excepciones que confirman la regla, como el caso de Colombia Compra Eficiente (11); sin embargo, infortunadamente no son muchas todavía. Por otra parte, los trabajadores, tanto en el sector público como en el privado, no han sido capacitados para dar respuestas rápidas e innovadoras ante circunstancias extraordinarias como la que representa la pandemia, sino más bien son temerosos de no seguir los complicados procesos vigentes que revisan las instituciones de control, o que siguen los modelos burocráticos que buscan destacar la importancia de un cargo (12).

Quisiera en este editorial dejar plasmados algunos de los problemas que ha significado llevar a cabo una investigación clínica durante la pandemia de la covid-19 en 2020. Esta investigación busca un tratamiento efectivo y seguro contra el SARS-CoV-2 (13), ya que hasta el momento de este escrito no disponemos de un tratamiento contra el virus o que controle la respuesta inflamatoria exagerada que desencadena la infección, más allá de la dexametasona, 
medicamento corticosteroide, que fue producido de manera sintética en 1958 para el tratamiento de la artritis reumatoidea (14) y de la cual se ha informado que reduce la mortalidad en un $30 \%$ en pacientes que requieren ventilación invasiva $(\mathrm{RR}=0,65$; IC $95 \%$ : 0,48-0,88) (15).

Los problemas para esta investigación se debieron a barreras que deberíamos superar, en el futuro próximo, para poder responder de manera adecuada a situaciones similares, en el campo de la investigación en salud. Vale la pena recordar que en los últimos 40 años hemos presenciado varias epidemias que han afectado de manera importante a nuestra población, entre ellas la del virus de inmunodeficiencia humana (VIH), descrito en 1981 (16); desde 2009 hemos tenido en América la aparición del virus H1N1 (17), al que siguió la aparición del chikunguña en 2014 (18), el zika en 2017 a partir de un brote originado en África en 2007 (19), con serias consecuencias en la salud infantil y, finalmente, la pandemia mundial por la covid-19 en 2020.

Es importante anotar que ante el avance de la pandemia, en abril de 2020 era prioritario iniciar el estudio lo más pronto posible, para poder ofrecer algunas alternativas de tratamiento a los pacientes, sobre todo los más críticos, y hacer una juiciosa evaluación de los beneficios y riesgos asociados. Estas barreras han llevado a un retraso de cerca de dos meses en la iniciación del estudio, tiempo que se habría podido ahorrar en beneficio de nuestros pacientes.

Los problemas identificados son:

1. La información y la desinformación.

Las revistas de investigación médica han sido consideradas como de alta credibilidad para la comunidad científica. Esta percepción ha estado basada en dos aspectos: la revisión por pares y los puntajes obtenidos en las métricas diseñadas para medir el desempeño de las revistas científicas. Los pares verifican la calidad científica y metodológica de los documentos publicados y las métricas describen el número de citaciones que recibe en promedio un artículo publicado en una revista específica (20). Sin embargo, tal vez por la necesidad de buscar una solución a una enfermedad nueva, con mal pronóstico para algunos grupos de pacientes, con un gran impacto sobre las unidades de cuidados intensivos por falla respiratoria e imprevistas consecuencias económicas y sociales, o por la tendencia que afecta a la prensa en general de querer ser quienes publican primero la noticia, se identificó que las revistas más importantes a nivel internacional empezaron a recibir una gran cantidad de manuscritos sobre la covid-19, que requirieron un proceso de revisión por pares simplificado (21); además, se empezaron a publicar estudios sin revisión por pares en sitios de preimpresos como bioRxiv y medRxiv (22). Lamentablemente, muchos de estos manuscritos tienen problemas de validez (23). Aquí vale la pena mencionar la premisa según la cual, en ciencias de la salud, se debe tener cautela con los beneficios de la transferencia rápida de nuevos conocimientos en relación con el potencial daño de estudios con alto riesgo de sesgos, que son publicados debido al proceso de revisión acelerado, con deficiencias metodológicas y en el informe final (24). Este problema de la desinformación y la publicación de estudios afectó localmente las investigaciones, como veremos más adelante.

2. La falta de experiencia en el manejo de situaciones de pandemia, que se evidenció en las decisiones de los comités de ética y de la comunidad médica en general.

En situaciones de pandemias virales no conocidas, los tratamientos se han utilizado con base en experiencias previas de infecciones similares en el pasado o los resultados in vitro de los medicamentos contra virus de la misma familia. De esta manera, a partir de los tratamientos utilizados en H5N1 y el MERS, al inicio de la epidemia se consideró el uso de hidroxicloroquina (25) y de lopinavir ritonvair (26) respectivamente. Basados en estos antecedentes, el consenso colombiano para el manejo de la covid-19 publicado en abril de 2020 recomendaba el uso de estos medicamentos, como también de la azitromicina (27). Sin embargo, 
en el mes de mayo se publica un estudio de Mehra et al. sobre la seguridad de la hidroxicloroquina, en el cual se presentaban los resultados de un estudio de "datos reales" en una cohorte de 96.032 pacientes en 671 hospitales, que mostró un mayor riesgo de muerte en pacientes que recibían hidroxicloroquina y aún mayor en la combinación hidroxicloroquina más azitromicina (28). La publicación de los resultados de este estudio en la revista Lancet, a pesar de proveer evidencia de baja certeza, motivó que de manera apresurada los comités de ética locales suspendieran los estudios que utilizaban estos medicamentos a nivel local, lo que conllevó atrasos en el proceso de iniciación del estudio. Además, el consenso colombiano también excluyó estos medicamentos a raíz de esta publicación (29). Vale la pena recordar que este estudio fue finalmente objeto de retractación por la revista, ya que no se pudo comprobar la veracidad de los datos (30). Esta actitud contrastó con la manera tranquila como el comité monitor del estudio Recovery, que se desarrolla en el Reino Unido, y que evaluaba también estos medicamentos, no modificó los brazos de tratamiento ante estos resultados, hasta que ellos tuvieran sus propias conclusiones. Es posible que los ingleses nos lleven alguna ventaja en el estudio y manejo de epidemias.

3. Contratación del personal de trabajo de campo en investigación.

En Colombia, desde hace largo tiempo, se utiliza la contratación de prestación de servicios, modalidad en la que el contratista es un trabajador independiente, que debe asumir el pago de sus prestaciones sociales (derechos de salud y pensión) y no hay relación laboral con la empresa que lo contrata (31). Además, el contratista debe asumir el costo de la afiliación al sistema de riesgos laborales en las categorías I, II y III (riesgo mínimo a medio), y la empresa está a cargo en el caso de los riesgos IV y V (alto o máximo). Bajo esta modalidad se ha contratado a los asistentes de investigación en Colombia en este periodo de pandemia. En el estudio en mención se requiere que los investigadores identifiquen pacientes con diagnóstico confirmado de covid-19 para explicarles el objetivo de la investigación y solicitar su participación mediante la firma de consentimiento informado. Dado que la infección tiene una tasa de ataque cercana al 12\% (32), los investigadores están en riesgo de adquirirla, para esto se les ha provisto de todo el kit de protección, que incluye aquel requerido en áreas en las que circulan aerosoles, con la intención de proteger a los investigadores y sus familias. Por estar en alto riesgo se hizo la consulta de qué pasaría con sus honorarios en caso de requerir aislamiento por adquirir la infección, a lo cual se nos indicó que se suspendería el contrato, por lo que quedarían sin honorarios. Al consultar el nivel de riesgo de la infección por covid-19 con las aseguradoras, se nos indicó que era nivel medio (III). Así, se buscó cubrir a los investigadores con un seguro de vida y de incapacidad laboral, para obviar la suspensión de los ingresos; sin embargo, ninguna entidad pública pudo cubrir este seguro. Esta situación llevó a la renuncia de varios de los asistentes de investigación, lo que demoró la inclusión de pacientes en algunas instituciones. Por otra parte, vale la pena anotar que el proceso de contratación en las entidades del Estado es muy lento, lleno de trámites, lo que hace que pueda demorar entre 15 y 30 días. En algunos casos, los procesos de contratación son contradictorios, por ejemplo, la entidad reguladora de medicamentos solicita la expedición del seguro de responsabilidad civil con todo el clausulado incluido y con fecha de expedición para la aprobación del protocolo, pero las entidades patrocinadoras no autorizan el pago con esa fecha por lo cual se requieren reprocesos que demoran la autorización para iniciar el estudio por parte de la entidad reguladora.

4. Los tiempos en las entidades participantes.

A pesar de que los comités de ética de las instituciones participantes y la entidad reguladora expresaron su disposición a gestionar las solicitudes de la manera más pronta posible, los hechos mostraron que a menudo son más importantes los trámites, que tener 
una respuesta ágil ante una pandemia de estas características. Como ejemplo tenemos que en el estudio se habían incluido la hidroxicloroquina y la azitromicina como medicamentos por evaluar, estos tienen como efecto adverso la prolongación del segmento QT en el electrocardiograma (EKG) (33), por lo que se excluían los pacientes que mostraban este signo en el EKG de tamización, por alto riesgo de muerte cardiaca. Debido a la evidencia de no efectividad de estos medicamentos y de la combinación lopinavir/ ritonavir que mostró el estudio Recovery $(34,35)$, fueron sustituidos por la emtricitabina/tenfovir, la colchicina y la rosuvastatina, que no tienen este efecto adverso. Mediante una carta se pidió a los comités de ética que se suspendiera su solicitud al ingreso ya que no eran requeridos y sí podrían demorar el inicio del tratamiento, hasta 24 horas, en pacientes que hubieran aceptado participar. Sin embargo, el comité de ética de una institución indicó que no se aprobaría dicho cambio hasta que no se sometiera la enmienda al protocolo. Es decir, primó el trámite sobre la relevancia del cambio para beneficio de los pacientes. Por otra parte, la entidad reguladora demoró cuatro semanas en dar la aprobación del inicio de la inclusión de pacientes en una institución. Para la fecha del envío de la solicitud había en Bogotá 201.919 infectados y 5.398 fallecidos, para la fecha de su expedición había 256.136 nuevos infectados y cerca de 6.753 fallecidos (36). El tiempo que tomó dar estas respuestas no es comprensible en una situación como la presente.

Este tiempo de pandemia nos ha llevado a acelerar procesos que se vislumbraban inviables a corto plazo, como el trabajo en casa basado en el uso masivo de las tecnologías de la comunicación y la información, o transformaciones no pensadas hace un año como el modo de relacionarnos con nuestros congéneres. Se requiere que el Estado, la sociedad como un todo y nosotros como seres individuales modifiquemos la rígida estructura mental que nos ata al subdesarrollo, e iniciemos una transformación individual y colectiva que nos permita aprender de nuestros errores para no repetirlos en el futuro y superar estas barreras, pues algunas veces parece que estuviéramos condenados a un destino invariable, en una paradoja similar a la planteada en la serie de televisión Dark (37).

Como sociedad, debemos ser capaces de responder de manera articulada en busca de la solución o, al menos, la atenuación del impacto de una crisis como la que enfrentamos, trabajando todos con un mismo objetivo para poder dar respuestas válidas y ágiles a situaciones extraordinarias como la actual, que nos permitan ser menos rígidos tanto en el tema de la contratación laboral como en la compra de bienes y servicios, o en el cumplimiento de trámites administrativos que nos llevan a la parálisis, pero también ser críticos con la avalancha de información que nos apabulla y que responde a veces a intereses comerciales o políticos que nos son ajenos. El no superar estas barreras será factor que perpetúe nuestro equívoco modelo de desarrollo.

Estas reflexiones seguramente son aplicables en otras áreas como: la atención en salud, la educación, la seguridad alimentaria, el trabajo, etc., que resultaron seriamente afectadas no solo por la importancia de la pandemia, por el miedo y la incertidumbre que la acompañan, sino también por la baja capacidad de respuesta y posiblemente inexperiencia de nuestra sociedad.

En la Misión de sabios del año 2019 se hablaba de las misiones emblemáticas que nos deberían unir como sociedad, como país; así, pues, la vida nos presenta un problema para unirnos y trabajar juntos por el bien de todos, por ejemplo, cómo garantizar la seguridad alimentaria de los niños de 2 a 5 años en este momento en el país. No perdamos esta oportunidad.

\section{Hernando Gaitán Duarte, MD, MSc.}

Editor

\section{REFERENCIAS}

1. Organización Mundial de la Salud. WHO DirectorGeneral's opening remarks at the media briefing on COVID-19. 11 March 2020. Disponible en: https:// 
www.who.int/dg/speeches/detail/who-directorgeneral-s-opening-remarks-at-the-media-briefing-oncovid-19---11-march-2020

2. Ghemawat P, Altman SA. The state of globalization in 2019, and what it means for strategists. Harvard Busissnes Review. 2019. Disponible en: https://hbr. org/2019/02/the-state-of-globalization-in-2019-andwhat-it-means-for-strategists

3. Mehra G. 7 ways that predictive analytics is transforming ecommerce. CXL Institute; 2020. Disponible en: https://cxl.com/blog/predictive-analytics-changingworld-retail/?hvid $=352 \mathrm{IDw}$

4. Ovum. The Future of E-commerce: The Road to 2026. Disponible en: https://www.criteo.com/es/wpcontent/uploads/sites/8/2017/09/ovum-the-future-ofe-commerce-the-road-to-2026.pdf

5. Radinsky K, Davidovich S, Markovitch S. Learning to predict from textual data. JAIR. 2012;45:641-84. https://doi.org/10.1613/jair.3865

6. Gates B. The next Outbreak. We are not ready. TED 2015. Disponible en: https://www.ted.com/talks/ bill_gates_the_next_outbreak_we_re_not_ready

7. Vera MF. Protesta y medios: ¿cubrimiento mediático o problema de enfoque? Centro de Investigación y Educación Popular (Cinep); 2019. Disponible en: https://www.cinep.org.co/Home2/component/ k2/750-protesta-y-medios-cubrimiento-mediaticoo-problema-de-enfoque.html

8. Bohorquez S. La movilización social marcaría la agenda de Colombia el próximo año, según Trust. La República. 2019, 20 de diciembre . Disponible en: https://wwwlarepublica.co/economia/la-movilizacionsocial-marcaria-la-agenda-de-colombia-el-proximoano-segun-trust-2946212

9. Transparency International. Report Corruption Colombia. Disponible en: https://www.transparency.org/ en/countries/colombia

10. Transparencia por Colombia: entre los países donde esfuerzos anticorrupción están estancados. 2020, 23 de enero. Disponible en: https://transparenciacolombia. org.co/2020/01/22/colombia-entre-los-paises-dondeesfuerzos-anticorrupcion-estan-estancados/
11. República de Colombia. Sistema de Compra Pública: Colombia Compra Eficiente; 2020. Disponible en: https://www.colombiacompra.gov.co/

12. Gaete Quezada R, Acevedo Muñoz S, Carmona Robles G, Palta Layana O. Generando buenas prácticas de innovación pública desde las regiones: "piensa con i". Innovar. 29(73):147-59. https://doi.org/10.15446/ innovar.v29n74.82095

13.Effectiveness and Safety of Medical Treatment for SARS-CoV-2 (COVID-19) in Colombia: A Pragmatic Randomized Controlled Trial. ClinicalTrials.gov identifier (NCT number): NCT04359095.

14. Cole J. The Cambridge Crystallographic Data Centre (CCDC). Dexamethasone briefing - Its chemistry and history; 2020. Disponible en: https://www.ccdc.cam. ac.uk/Community/blog/dexamethasone/

15. University of Oxford. Low-cost dexamethasone reduces death by up to one third in hospitalised patients with severe - The Recovery Trial. Oxford. 2020;1-4.

16. CDC. Pneumocystis pneumonia - Los Angeles. MMWR Weekly. 1981;30(21):1-3. https://www.cdc. gov/mmwr/preview/mmwrhtml/june_5.htm

17. Núñez LM, Aranda DF, Jaramillo AC, Moyano LF, Osorio EJ. Cronología de una pandemia: la nueva Influenza A H1N1 en Bogotá, 2009-2010. Rev Salud Pública. 2011;13(3):480-91.

18. Organización Panamericana de la Salud (OPS), Organización Mundial de la Salud (OMS). Alerta Epidemiológica. Fiebre por Fiebre por chikungunya; 2013. Disponible en: http://www.paho.org/hq/ index.php?option $=$ com_docman\&task $=$ doc $_{-}$ view\&gid=23807\&Itemid. 2013:5

19. Weaver SC, Costa F, Garcia-Blanco MA, Ko AI, Ribeiro GS, Saade G, et al. Zika virus: History, emergence, biology, and prospects for control. Antiviral Res. 2016;130:69-80. https://doi.org/10.1016/j. antiviral.2016.03.010

20. Bradshaw CJ, Brook BW. How to Rank Journals. PLoS One. 2016;11(3):e0149852. https://doi.org/10.1371/ journal.pone.0149852.

21. Bauchner H, Fontanarosa PB, Golub RM. Editorial. Evaluation and peer review during a pandemic: How 
journals maintain standards. JAMA. 2020;324(5):4534. https://doi.org/10.1001/jama.2020.11764

22. Škorić L, Glasnović A, Petrak J. A publishing pandemic during the covid-19 pandemic: How challenging can it become? Croat Med J. 2020;61(2):79-81. https:// doi.org/10.3325/cmj.2020.61.79.

23. Jarvis C. Journals, peer reviewers cope with surge in covid-19 Publications. The Scientists; 2020. Disponible en: https://www.the-scientist.com/newsopinion/journals-peer-reviewers-cope-with-surge-incovid-19-publications-67279

24. Wolkewitz M, Puljak L. Methodological challenges of analysing covid-129 data during the pandemic. BMC Med Res Methodol. 2020;20:81. https:/doi. org/10.1186/s12874-020-00972-6

25. Devaux CA, Rolain J-M, Colson P, Raoult D. New insights on the antiviral effects of chloroquine against coronavirus: What to expect for covid-19? Int J Antimicrob Agents. 2020:105938. https://doi.org/10. 1016/j.ijantimicag.2020.105938

26. Mo Y, Fisher D. A review of treatment modalities for Middle East respiratory syndrome. J Antimicrob Chemother. 2016;71:3340-50. https://doi.org/ 10. 1093/jac/dkw338

27. Asociación Colombiana de Infectología (ACIN). Consenso Colombiano de atención, diagnóstico y manejo de la Infección por Sars CoV-2/Covid-19 en establecimientos de atención de la salud. Infectio. 2020;24(3).

28. Mehra MR, Desai SS, Ruschitzka F, Patel AN. Hydroxychloroquine or chloroquine with or without a macrolide for treatment of covid-19: A multinational registry analysis. Lancet. 2020;22:S0140-6736(20) 31180-6. https://doi.org/10.1016/S0140-6736 (20) 31180-6

29. Asociación Colombiana de Infectología. Recomendaciones para el manejo de infección por Sars CoV-2-Covid-19. Bogotá: ACIN; 2020. Disponible en: https://www.acin.org/images/guias/coronavirus/ Comunicado_10.pdf
30. Mehra MR, Ruschitzka F, Patel AN. RetractionHydroxychloroquine or chloroquine with or without a macrolide for treatment of COVID-19: A multinational registry analysis. Lancet. 2020;395(10240):1820. https://doi.org/10.1016/S0140-6736(20)31324-6

31. Roldán JA. Regulación del contrato de prestación de servicios. Asuntos Legales. 2014. Disponible en: https:// www.asuntoslegales.com.co/consultorio/regulaciondel-contrato-de-prestacion-de-servicios-2168926

32. Jing QL, Liu MJ, Zhang ZB, Fang LQ, Yuan J, Zhang AR, et al. Household secondary attack rate of covid-19 and associated determinants in Guangzhou, China: A retrospective cohort study. Lancet Infect Dis. 2020;20(10):1141-50. https://doi.org/10.1016/ S1473-3099(20)30471-0

33. Borba MGS, Val FFA, Sampaio VS, et al. Effect of high vs low doses of chloroquine diphosphate as adjunctive therapy for patients hospitalized with severe acute respiratory syndrome coronavirus 2 (SARS-CoV-2) infection: A randomized clinical trial. JAMA Netw Open. 2020;3(4):e208857. https://doi.org/10.1001/ jamanetworkopen.2020.8857

34. Recovery Trial. No clinical benefit $t$ from use of hydroxychloroquine in hospitalised patients with. 2020;5-7.

35. RECOVERY Trial. No clinical benefit from use of lopinavir-ritonavir in hospitalised COVID-19 patients studied in RECOVERY. 2020;1.5. Disponible en: https:// www.recoverytrial.net/news/no-clinical-benefit-fromuse-of-lopinavir-ritonavir-in-hospitalised-covid-19patients-studied-in-recovery

36. Instituto Nacional de Salud Colombia. Covid 19 en Colombia. Bogotá: El Instituto; 2020. Disponible en: https://www.ins.gov.co/Noticias/Paginas/coronavirusdepartamento.aspx

37. Ortiz M. Serie Dark. Cultura genial. Disponible en: https://www.culturagenial.com/es/serie-dark/ 